\title{
THE PERMEABILITY OF ARTICULAR CARTILAGE
}

\author{
A. Maroudas, London, P. Bullough, Oxford, S. A. V. Swanson and M. A. R. Freeman, \\ LONDON, ENGLAND
}

In the course of a wider study of human articular cartilage with special reference to the development of osteoarthritis, some of the mechanisms of normal nutrition and fluid exchange in articular cartilage have been investigated.

Because it has been suggested that after death there is a rapid fall in the diffusion rate of substances into cartilage (Brower, Akahoshi and Orlic 1962), the first part of this study deals with a comparison between diffusion rates of dye into cartilage in vivo and in vitro.

In the second part some preliminary results on the diffusion rates of different solutes into cartilage are presented. The effect of agitation of the fluid in which cartilage is immersed upon the rate of diffusion of substances is discussed. Permeabilities from both the articular surface and the subchondral bone have been investigated in an attempt to determine which of the two routes is the more important in the nutrition of cartilage. A sample calculation is given to illustrate the applicability of these results to an understanding of cartilage nutrition.

Finally, the suggestion that some "pumping action" is important in the nutrition of cartilage is examined both experimentally and theoretically.

\section{COMPARISON OF DIFFUSION RATES IN VITRO AND IN VIVO}

Up to now most of the work on the permeability of cartilage has been done in vivo. Most of the results have been inconclusive and none of them has been quantitative. In order to obtain a completely integrated picture of the functioning of the living joint, the ideal experiment should be carried out under the complex conditions of use. However, as a first

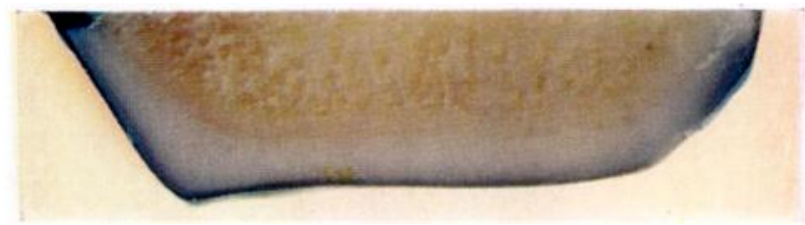

FIG. 1

A typical slice showing dye penetration into cartilage.

step, it is necessary to isolate the variables involved and examine their effects separately. It would be difficult to follow this procedure in a living animal. Hence it has been the object of the present study to carry out the experiments in vitro, using as far as possible apparatus and methods capable of giving objective, quantitative and unambiguous results. An added advantage of working in vitro is that it is possible to use human material.

In order to deal with the objection raised by the work of Brower, Akahoshi and Orlic (1962) a comparison was made between the rates of dye penetration in cartilage: 1) in a living animal; 2) immediately after death; 3) four hours after death; and 4) twenty-four hours after death. The pig was chosen as the experimental animal because its cartilage is comparable in thickness with human cartilage.

A preliminary set of experiments was carried out on rabbits, but, although our results were in qualitative agreement with the later results obtained in pigs, the cartilage was so thin that it was found to be very unsuitable for making accurate measurements. 
EXPERIMENTAL PROCEDURE

Three pigs approximately five months old and weighing 35 kilograms were used. The knee joint was chosen for dye injection in vivo and a procedure was adopted which permitted the study of dye penetration into cartilage in one mobile and one immobile joint in the same pig.

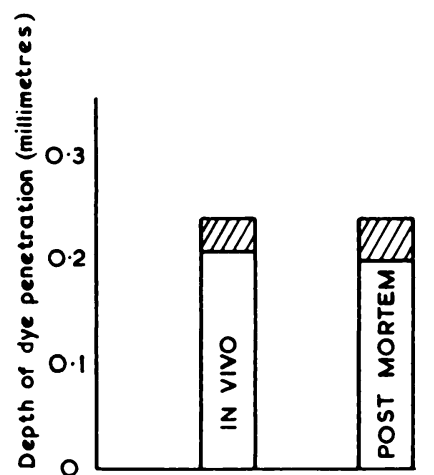

FIG. 2

Comparison between dye penetration into cartilage in vivo and after death in the absence of agitation.

Time of contact with dye: fortyfive minutes.

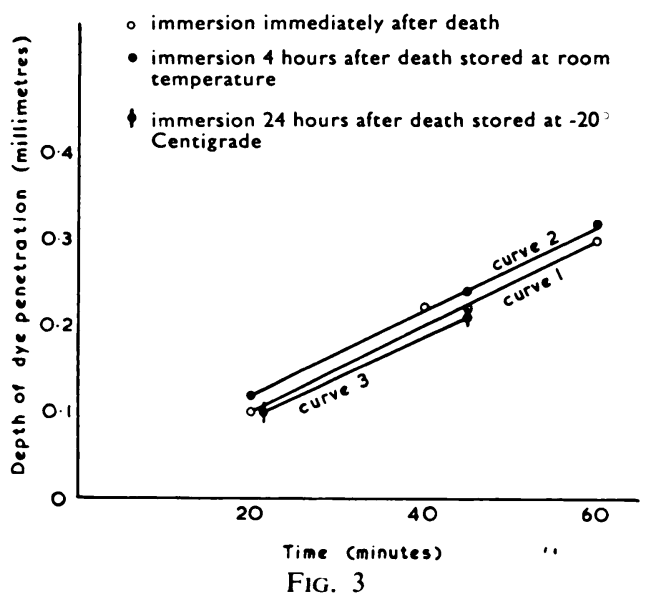

Variation in the depth of dye penetration with time in vitro.

The pig was given a general anaesthetic, and 10 millilitres of a $0 \cdot 1$ per cent solution of methylene blue in normal Ringer's solution was injected into one knee joint (joint A). After recovery from anaesthesia the pig was allowed to walk. After approximately half an hour the pig was anaesthetised again, the leg containing joint $A$ was amputated under a tourniquet and joint $A$ was excised for examination. Dye solution was then injected into the second knee joint (joint B), but this time the pig was left under anaesthesia, completely immobile. It was killed after approximately half an hour and joint B was removed for examination. Both joints remained in contact with the dye for the same period of time.

Ankle and hip joints were excised immediately upon amputation or death and were used to study dye penetration in vitro. Part of the cartilage was used immediately after death, part was kept for four hours unfrozen and part was kept for twenty-four hours at -20 degrees Centigrade. In each case dye penetration was studied by immersing the cartilage in a beaker containing a $0 \cdot 1$ per cent solution of methylene blue in Ringer's solution for periods ranging from twenty minutes to one hour. In one set of experiments the solution of dye in the beaker was left unstirred, and in the other vigorous agitation was provided.

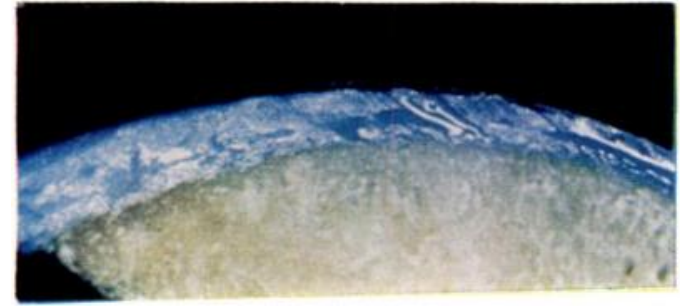

FIG. 4

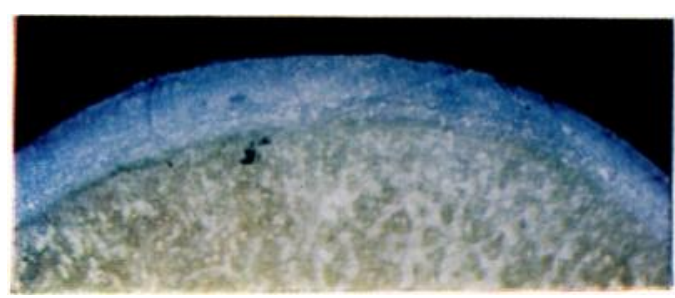

Fig. 5

Slices of cartilage which had been immersed in dye solution for forty-five minutes. Figure 4-Immediately after death. Figure 5-Four hours after death.

In order to estimate the rate of dye penetration, sections of cartilage about 1 millimetre in thickness were cut normal to the joint surface and were examined under a dissecting microscope provided with a graticule. The depth of penetration was noted in each case, as 
measured by the width of the dark blue band extending from the surface of the cartilage (Fig. 1). Three readings per slice were taken and three slices were used for each determination.

\section{RESULTS AND DISCUSSION}

The results are presented graphically in Figures 2 and 3. Figure 2 shows a comparison between the depth of dye penetration into cartilage in vivo in the immobilised joint and in vitro

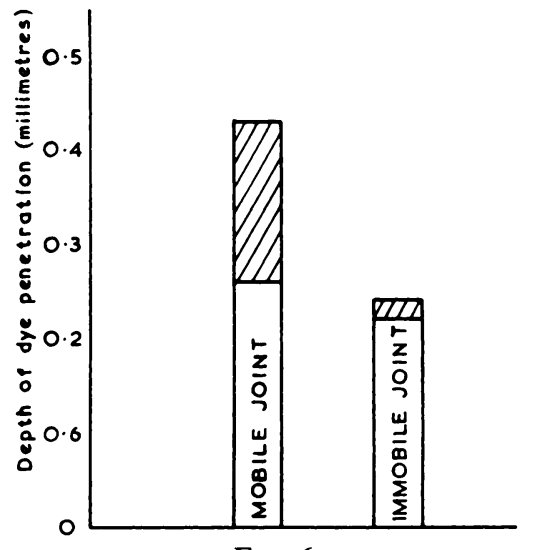

Fig. 6

Comparison between dye penetration in vivo in a mobile and immobile joint. in the absence of stirring. It can be seen that the rate of dye penetration is the same in both cases. The hatched sections represent the experimental scatter. Figure 3 shows quantitatively the variation of the depth of dye penetration into cartilage with the time of exposure to the dye in vitro at various times after death. It can be seen that the depth of dye penetration into cartilage varies in a linear manner with the time of exposure to the dye and that the curves for cartilage tested immediately after death (curve 1), four hours later (curve 2) and twenty-four hours later (curve 3) practically coincide. If there were a drastic decrease in the rate of diffusion within the first hour after death, as was claimed by Brower and colleagues, curve 1 would not be a straight line but would be concave downwards, and it would not coincide with curves 2 and 3 .

Figures 4 and 5 show photographs of two of the slices of cartilage on which measurements had been made. Slices $A$ and $B$ were taken from cartilage which had been immersed in stirred dye solution for forty-five minutes, immediately after death, and four hours after death respectively.

In an independent set of experiments carried out on human necropsy specimens it has also been shown that storage at -20 degrees Centigrade for days or even weeks does not affect the rate of diffusion of substances into cartilage.

It can thus be concluded that material transfer into cartilage is subject to the normal physico-chemical processes of diffusion; that is to say, cartilage does not exhibit any transport properties dependent on the activity of living cells. It may further be said that no significant changes liable to affect material transfer occur in the matrix of cartilage after death, provided that the cartilage is kept at a suitable low temperature ( -20 degrees Centigrade). Thus studies based on cartilage obtained from necropsy specimens are valid.

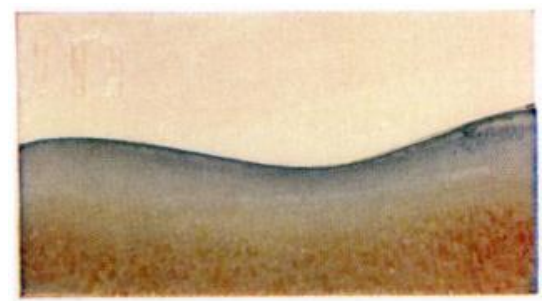

Fig. 7

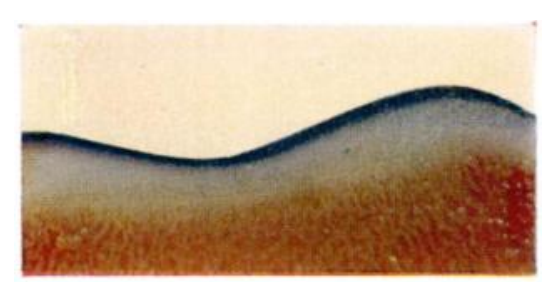

FIG. 8

Effect of joint mobility on rate of dye penetration in vivo. Figure 7-Mobile joint. Figure 8-Immobile joint.

The effect of joint mobility in vivo on the rate of dye penetration is shown in Figures 6 and 7. Figure 6 shows a graphical comparison between dye penetration in vivo in a mobile and in an immobile joint. It can be seen that the rate of diffusion is significantly lower in the immobile joint. This effect will be discussed later. Figures 7 and 8 show photographs of two of the slices of cartilage on which the measurements given in Figure 6 are based. The fact that the colour intensity is lower in the mobile joint is presumably due to the escape of dye through the synovial membrane. 


\section{DIFFUSION RATES OF SUBSTANCES INTO CARTILAGE IN VITRO}

Since the experiments reported above indicate that the permeability of living and dead cartilage is similar, all further diffusion experiments were carried out in vitro.

\section{APPARATUS AND EXPERIMENTAL PROCEDURE}

Necropsy specimens of human cartilage taken from the femoral condyles were used in all experiments. In most of the diffusion experiments thin $(200 \mu)$ slices about 1 square centimetre in area were cut on a sledge microtome. Each slice was of uniform, accurately known thickness and was used as a membrane in a specially designed diffusion cell.

In the experiments in which the diffusion of substances through the subchondral bone was investigated, slices cut to include the subchondral bone, the calcified zone (when present) and about 0.5 millimetre of overlying cartilage were employed.

The diffusion cell used is shown in Figure 9. It consisted of two compartments separated by the cartilage membrane. Both compartments contained Ringer's solution and provision was made for stirring. The solute diffused across the membrane and samples were periodically withdrawn for analysis.

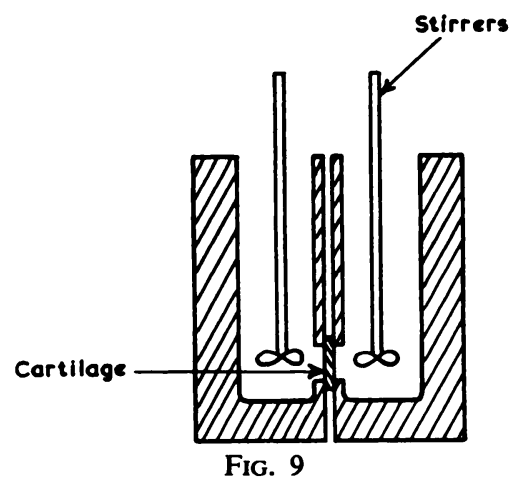

Diffusion cell. Sectional side view.

Whereas the visual observation of the depth of dye penetration into cartilage is only an approximate method of estimating the rates of diffusion, the diffusion cell is capable of giving accurately quantitative results.

\section{THEORY}

It may be helpful at this stage to consider briefly what happens when a solute is transferred from one phase to another across an interface.

In the case to be considered here the two phases were 1) the solution and 2) a membrane, namely cartilage. Let it be assumed that a solute $\mathrm{A}$ is initially present in the fluid and absent in the membrane. If the concentration of $A$ were uniform in the fluid right up to the interface, the rate of transfer of $A$ into the membrane would be dependent only on the concentration gradient of $\mathrm{A}$ across the membrane and on the diffusion coefficient of $\mathrm{A}$ in the membrane. The diffusion coefficient is a measure of the mobility of molecules of a species in a given medium.

In equation form this would be written as:

$$
\mathrm{J}_{\mathrm{A}}=-\mathrm{D} \frac{d \mathrm{C}}{d x},
$$

where $J_{A}=$ flux of molecules of $A$, moles per second per square centimetre of surface,

$\mathrm{D}=$ diffusion coefficient of $\mathrm{A}$ in the membrane, square centimetres per second, $\frac{d \mathrm{C}}{d x}=$ concentration gradient of $\mathrm{A}$ in the membrane, moles per cubic centimetre per

Equation (la) is known as Fick's law.

If the concentration gradient across the membrane is linear and the concentration of $\mathrm{A}$ in the fluid on one side of the interface is $\mathrm{C}=\mathrm{C}_{i}$ and on the other side is $\mathrm{C}=0$, equation (1a) can be written as:

$$
\mathrm{J}_{\mathrm{A}}=\mathrm{D} \frac{\mathrm{C}_{i}}{h},
$$

where $h=$ membrane thickness.

In practice the region of the fluid close to the interface, within the stagnant film, soon becomes depleted of A and a concentration gradient therefore develops in the fluid. The

Vol. 50 B, NO. 1, FEBRUARY 1968

$\mathbf{L}_{1}$ 
molecules of A, apart from having to diffuse through the membrane, now have to make their way towards the membrane through a film of the solution. Therefore, in the absence of stirring, both the membrane and the liquid offer a resistance to material transfer and affect the overall rate of diffusion of A into the membrane (Fig. 10). If the fluid is vigorously stirred it is possible to maintain a uniform concentration of A throughout the bulk of the fluid phase, although there inevitably remains a more or less thin, stagnant, liquid film adhering to the interface (Fig. 11). The more efficient the stirring, the thinner the stagnant film and the lower the resistance of the liquid phase to the transfer of $A$. If stirring is adequate the resistance of the liquid film is usually small compared with the resistance of the membrane.

Equation (lb) strictly applies to systems in which the resistance to diffusion is present in the membrane only, and where the membrane is non-selective to different ions. These conditions are very rarely achieved in practice. Hence the quantity which is determined experimentally is not the diffusion coefficient $D$, but an overall permeability coefficient $P$ which is defined by a modified form of equation ( $1 \mathrm{~b})$, namely:

$$
\mathbf{J}_{\mathbf{A}}=\mathbf{P} \frac{\text { Co }}{h},
$$

where $\mathrm{Co}$ is the concentration of $\mathrm{A}$ in the bulk of the solution, moles per cubic centimetre, $\mathbf{P}=$ Permeability coefficient of $\mathbf{A}$, square centimetres per second, as before,

$\mathbf{J}_{\mathbf{A}}=$ flux of molecules of $\mathrm{A}$, moles per second per square centimetre of surface.

\section{RESULTS AND DISCUSSION}

Permeability to electrolytes and non-electrolytes-Figure 12 shows the permeability of normal adult human cartilage at room temperature, with and without stirring, to methylene blue, glucose and alizarin red. Fuller information about diffusion coefficients and their variation in relation to age, degenerative change and depth from the synovial surface will be presented elsewhere (Maroudas 1968).

The permeability coefficient of methylene blue is about three times as high as that of glucose, which in turn is twice that of alizarin red. This result can most probably be explained by two factors: 1 ) the great affinity which the ground substance of cartilage shows for methylene blue; and 2) the fact that cartilage is a membrane possessing a preponderance of fixed negative charges due to the anionic groups-sulphate and carboxylate-of the chondroitin sulphate. The passage of cations through such negatively charged membranes is usually somewhat faster than that of non-electrolytes, which in turn is faster than that of anions, provided the molecular sizes are approximately the same.

The finding that all three types of substance penetrate into cartilage, but at slightly different speeds, is in accordance with physico-chemical principles and also with the fact that different kinds of solutes including anions-such as sulphate-are known to become incorporated into cartilage in life. However, Kantor and Schubert (1957) claimed on experimental grounds that no anionic dyes diffused into cartilage. Our work does not confirm this finding. Our results may differ from those of Kantor and Schubert because they used dyes in water and not in salt solution (Helfferich 1962). Thus, Kantor and Schubert's results are not applicable to physiological conditions.

The effect of stirring-The values of the permeability coefficients obtained with stirring are two to four times as high as those obtained without stirring. (Alizarin red was only examined stirred.) This finding is in accordance with theory, and since joint movement is the only method by which synovial fluid may be agitated in life it suggests that joint movement may play an important part in the nutrition of articular cartilage. As described above, it was indeed found that in the mobile joint of the living animal dye diffusion proceeded at a higher rate than in the immobile joint. 
The values obtained without stirring showed considerable scatter (Fig. 13). This result was to be expected since in the absence of regular stirring there is always some convection resulting in a slight but variable degree of agitation. Thus reproducible values for the diffusion rates can only be obtained if vigorous stirring of the solutions on both sides of the membrane is ensured.

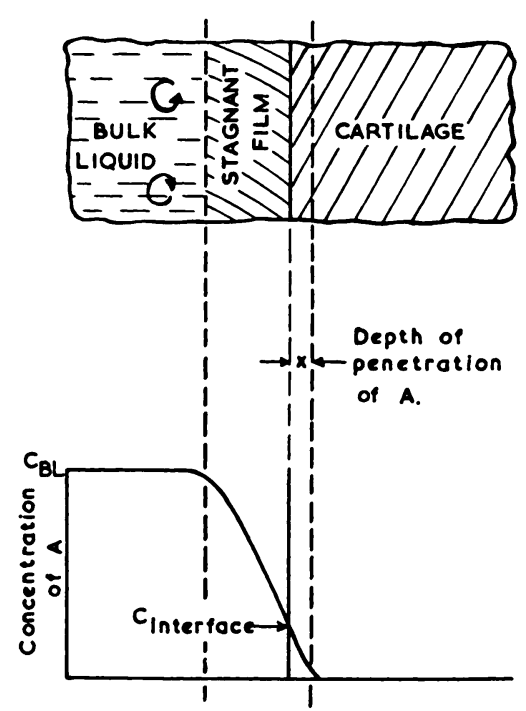

Fig. 10

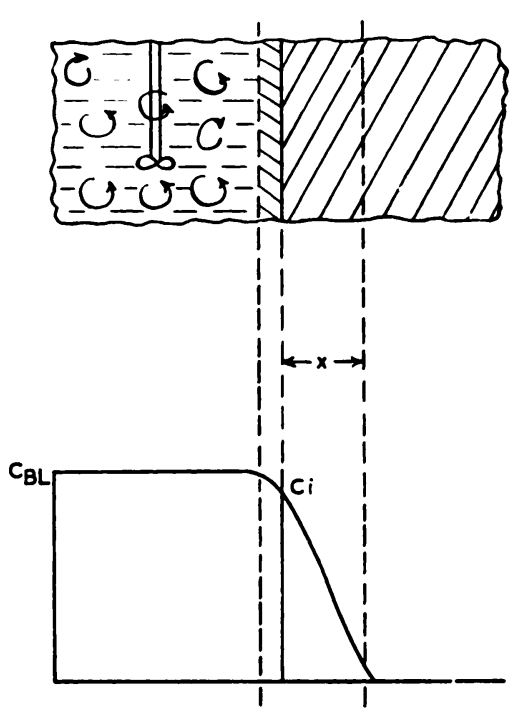

Fig. 11

Figure 10-Penetration of cartilage by molecules with little stirring of the fluid. Figure 11-Penetration of cartilage by molecules with vigorous stirring.

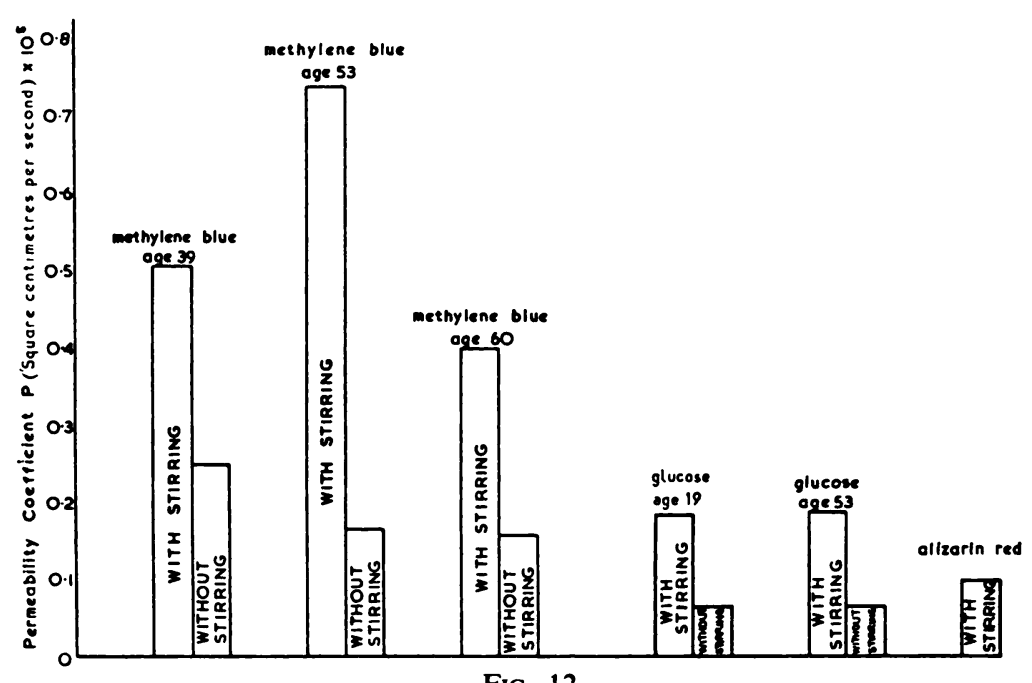

FIG. 12

Mean permeability coefficients for methylene blue, glucose and alizarin red in cartilage obtained with and without stirring.

PERMEABILITY OF THE BONE CARTILAGE INTERFACE

Nutrients presumably reach chondrocytes either through the synovial surface of cartilage or from the subchondral bone, or by both routes. There can be no doubt, both from the work reported in this study and from that of others, that the synovial surface is permeable to water and solutes such as glucose. On the other hand the literature does not contain unequivocal evidence on the question of the permeability of the bone-cartilage interface. 
Collins (1949) stated that the calcified zone of cartilage constituted an impermeable barrier to the transport of material and believed that nutrients reached cartilage only through the articular surface. Ekholm $(1951,1955)$ considered that nutrition of cartilage occurred through

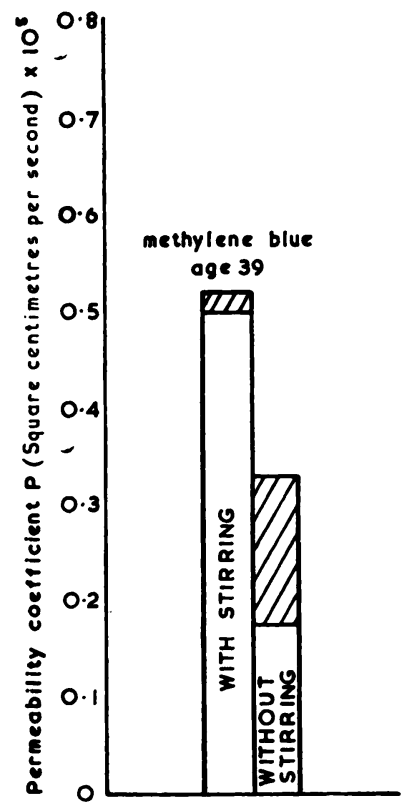

Fig. 13

Scatter in the values of the permeability coefficient with and without stirring. both routes. Brower and colleagues (1962), in experiments on both immature and adult rabbits, observed no penetration from the subchondral bone. On the other hand McKibbin and Holdsworth (1966) recently reported that in lambs growing cartilage derives a significant part of its nutrition from the underlying bone.

In view of the contradictory evidence we have studied the permeability of the bone-cartilage interface in adult human postmortem specimens and to a limited extent in immature cartilage. We found within the limits of our experimental methods that neither glucose, dye nor water passed across specimens of cartilage and the underlying bone taken from the femoral condyles of nineteen-, thirty-nine-, fifty-three-, fifty-eight- and sixty-yearold human adults. On the other hand specimens taken from the joint of a ten-year-old child were found to be permeable to water, glucose and methylene blue. Because of the shortage of material, no other immature human specimens were tested. However, it was observed in experiments carried out on immature pigs that methylene blue diffused across the bone-cartilage interface.

Thus our experiments show that mature cartilage can exchange material at a measurable rate through the articular surface only, whereas immature cartilage can obtain nutrients from the bone as well as from the joint cavity. This, taken together with our observations on the effect of stirring and joint movement, may have some bearing on the clinical observation that stiffness is not a problem after the immobilisation of a child's joint whereas it is a problem in the adult.

Experiments are in progress at present to establish the nature of the barrier to material transfer at the bone-cartilage interface and the results will be reported elsewhere.

\section{AVAILABILITY OF GLUCOSE IN THE BASAL LAYERS OF ADULT CARTILAGE}

Given that mature cartilage derives its nutrition chiefly from the joint cavity, and knowing the permeability coefficient of glucose, it is possible to calculate the thickness of cartilage which can adequately be supplied with this substance.

A simplified model is used in which it is assumed that the rate at which glucose is consumed by the cells is independent of the glucose concentration in the matrix and that the cells are capable of extracting all the glucose in the matrix. According to this model the concentration of glucose in the cartilage will decrease in a linear manner with the distance from the articular surface, reaching zero at a depth where the rate of diffusion of glucose from the surface is exactly balanced by the rate of consumption. This depth can be calculated from an integrated form of Fick's law. It can readily be shown that the final expression for the maximum depth of penetration of glucose is:

$$
d=\frac{2 \mathrm{PCo}}{\mathrm{Q}}
$$

where $\mathbf{P}=$ Permeability coefficient, square centimetres per second,

$\mathrm{Co}=$ Concentration of glucose in the synovial fluid, grammes per cubic centimetre,

$\mathrm{Q}=$ Rate of consumption of glucose by cartilage, grammes per cubic centimetre per second. 
RESULTS

From Figure 9 the value of the permeability coefficient for glucose at 20 degrees Centigrade is $\mathrm{P}=2.0 \times 10^{-6}$ square centimetre per second in the presence of stirring, and $\mathrm{P}=0.8 \times 10^{-6}$ square centimetre per second without stirring. Since it is possible to assume (Perry 1950) that $\mathbf{P}$ varies directly with the absolute temperature and inversely with the viscosity of the medium, the value of $P$ at body temperature has been estimated to be $2.9 \times 10^{-6}$ square centimetre per second with stirring and $0.55 \times 10^{-6}$ square centimetre per second without stirring.

Bywaters (1937) quoted $\mathrm{Q}=0.2$ mill gram per cubic centimetre per hour. $\mathrm{Co}=0 \cdot 8$ milligram per cubic centimetre (Ropes and Bauer 1953).

When the above figures are substituted into equation (3), the following values for $d$ are obtained: with stirring $d=3$ millimetres, without stirring $d=1.7$ millimetres.

DISCUSSION

Bywaters (1937), using a formula similar to equation (3) and an assumed value for $\mathrm{P}$, calculated $d$ to be equal to 3 millimetres. It is remarkable that his value agrees so closely with the value based on an experimentally obtained value of $P$.

In most human joints the thickness of cartilage does not exceed 3 millimetres, so that under normal conditions of joint movement producing agitation of the synovial fluid the cells should have an adequate supply of glucose. However, without agitation $d=1.7$ millimetres in Ringer's solution. Ogston and Sherman (1961) reported that in synovial fluid the diffusion coefficient of glucose was 25 per cent lower than in pure buffer. Thus in the presence of stagnant synovial fluid one might expect $d$ to be as low as 1.5 millimetres. This figure suggests that serious nutritional deficiencies may arise for chondrocytes deep in articular cartilage when an adult human joint is immobilised-a finding which emphasises the important part played by joint movement in cartilage nutrition. It may be that the changes reported in immobilised cartilage in the rat by Evans, Eggers, Butler and Blumel (1960) can partly be explained on this basis.

\section{THE ROLE OF ALTERNATE COMPRESSION AND RELAXATION IN CARTILAGE NUTRITION}

In previous sections of this paper consideration has been given to the transport of solutes by diffusion only. It has been suggested, however, that cartilage may derive its nutrition from the action of a physiological pump by which the compression of cartilage surfaces under load leads to expression of fluid, whilst the subsequent relaxation is accompanied by the absorption of fresh fluid. This suggestion has been examined both experimentally and theoretically.

\section{EXPERIMENTAL STUDY}

A femoral head obtained from a human necropsy specimen was immersed in a solution of methylene blue dye in Ringer's solution and inserted in an apparatus designed to test the compressibility of cartilage (Kempson, Swanson and Freeman 1967). A small area of cartilage was then subjected to one-minute periods of indentation under a pressure of 400 pounds per square inch, followed by one-minute recovery periods during which the indentor was removed from the surface. After half an hour the experiment was stopped, the femoral head was taken out of the dye solution, and slices of cartilage cut normal to the surface were removed: 1) from the area which had been subject to loading; and 2) from the unloaded areas which had been immersed in the dye. The dye solution was not systematically stirred, although some agitation was provided by the movement of the indentor head. The depth of dye penetration was measured by the method already described. No difference was found between the area subject to compression, where " pumping" might have occurred, and the unloaded areas where only diffusion could have been operative.

VOL. $50 \mathrm{~B}$, NO. 1, FEBRUARY 1968 


\section{THEORETICAL CONSIDERATIONS}

When part of the articular surface of cartilage is placed under load, fluid will be forced out of this zone in a radial direction since the subchondral bone end-plate is impermeable. An expression for radial flow through cartilage which can approximately be applied to describe the above situation was given by McCutchen (1962) in the following form:

$$
\frac{d h}{d t}=\frac{8 p \delta_{t} h}{\mathbf{R}^{2}}
$$

where $h=$ cartilage thickness,

$$
\begin{aligned}
\frac{d h}{d t} & =\text { rate at which cartilage is diminishing in thickness whilst under load, } \\
\mathrm{R} & =\text { radius of cartilage area under load, } \\
p & =\text { average pressure applied to cartilage under load, } \\
\delta_{t} & =\text { tangential permeability. }
\end{aligned}
$$

McCutchen neglected the deformation of the matrix itself and assumed that $\frac{d h}{d t}=$ rate of expression of fluid from cartilage. This assumption is acceptable at low loads, but work at present in progress in this laboratory shows that at higher loads significant deformation of the cartilage occurs in a time too short to be accounted for by the expression of fluid (Kempson et al. 1967). The subsequent thinning of cartilage is, as McCutchen assumed, chiefly due to the expression of fluid. It is thus possible to use McCutchen's equation, but to take the slope of the curve after the instantaneous response as the rate of expression of fluid.

In equation (4) the value of the tangential permeability coefficient, $\delta_{t}$, is assumed constant and independent of load. This assumption holds at low loads, but at high loads the permeability coefficient is considerably decreased since the greater the load the greater will be the degree of elastic deformation of the matrix and consequently the lower its permeability to liquid flow. Thus it is necessary to use in equation (4) the value of the permeability coefficient obtained for cartilage under the specified load $\left(\delta^{\prime}{ }_{t}\right)$.

\section{CALCULATION}

In order to calculate by means of equation (4) the rate at which fluid is displaced from cartilage under load it is necessary to know the load applied as well as the area over which it acts. Neither of these quantities is known precisely. However, most authors appear to agree that in the hip joint the ratio of maximum force during walking to body weight can be taken as about two and a half times (Inman 1947, Denham 1959, Rydell 1966). On the basis of the geometry of cartilage surfaces in the hip joint it will be assumed that the area over which the load acts is about 1 square inch. The average load would thus be about 400 pounds per square inch.

An approximate value for the permeability coefficient of cartilage subjected to a load of 400 pounds per square inch $\delta^{\prime}{ }_{i},(400)$ was obtained from the slope of the deformation curves for the head of the femur of human post-mortem specimens (Kempson et al. 1967). An average value of the slope was taken over the four-minute period starting one minute after the application of load. The permeability coefficient was calculated to be $\delta^{\prime}{ }_{t},(400)=6 \times 10^{-14}$ cubic centimetres per second per gramme.

The average thickness of cartilage was taken as $h=2.5$ millimetres. Thus in consistent units, $p=27.6 \times 10^{-6}$ gramme per centimetre per square second,

$\delta_{t}=6 \times 10^{-14}$ cubic centimetre second per gramme,

$\mathbf{R}=0.5$ centimetre,

$h=0.25$ centimetre,

Substitution of these values into equation (4) gives

$\frac{d h}{d t}=1 \cdot 2 \times 10^{-4}$ centimetre per minute. 
Thus the rate at which fluid is expelled is given by

$q=1 \cdot 2 \times 10^{-4}$ cubic centimetre per square centimetre per minute.

If the compression and the relaxation periods are approximately of the same duration the overall rate of imbibition of fluid (which in life can be assumed to be solute-rich) will thus be given by

$$
\frac{q}{2}=6.0 \times 10^{-5} \text { cubic centimetre per square centimetre per minute. }
$$

For a solute with a concentration in the fluid of 0.1 gramme per 100 cubic centimetres (e.g., glucose) the total weight of the solute transferred into cartilage is thus $6.0 \times 10^{-8}$ gramme per square centimetre per minute.

The amount of solute transferred by diffusion can be calculated from equation (2). Let the concentration of the solute $\mathrm{Co}=0 \cdot 1$ gramme per 100 millilitres at the surface of the cartilage and let $\mathrm{C}=0$ at a distance of, say, $x=2.5$ millimetres from the surface. Assume a linear concentration gradient and a value for the permeability constant $\mathrm{P}=2 \times 10^{-6}$ square centimetre per second. Substitution of these values into equation (2) gives $J_{A}=4.8 \times 10^{-7}$ gramme per square centimetre per minute, which is much higher than the amount of solute transferred by the action of a physiological pump, as calculated above.

However, peak loads in the load-bearing joints may in fact be higher than the average figure of $\mathbf{4 0 0}$ pounds assumed in our calculation. The area over which the load is distributed may also be lower. Both these factors would tend to increase the importance of the pumping mechanism. It is unlikely, however, that the increase would be such that the amount of solute introduced with the fresh liquid would exceed that provided by normal diffusion from the joint cavity in the absence of loading.

While pumping is taking place, free diffusion of solutes from the joint cavity into the loaded area must be considerably decreased because: 1) during the loading part of the cycle cartilage under load is no longer in immediate contact with a pool of solute-rich solventthat is, synovial fluid; and 2) compression of the cartilage will reduce its permeability to solutes. Thus the higher the load the lower the rate of transport of solutes by diffusion. Conversely, the higher the load, the higher the rate of transport of solutes by the action of the pump. The two transport mechanisms thus appear to complement each other. At high pressures the pump may contribute a substantial share to the transport of substances into cartilage. At low pressures the part played by the pump is insignificant. It must be stressed that under no likely circumstances could the physiological pump be expected to increase the rate of transport above that which could be achieved by simple diffusion.

The pump will operate provided the cartilage is loaded intermittently, either by moving the joint or by intermittently removing the load. If the periods of loading are prolonged, transport of solutes by diffusion will be greatly reduced for the reasons stated above, but at the same time relatively little fresh fluid will be pumped into cartilage. Under these conditions the overall rate of transfer of substances into cartilage will be very low. Immobilisation can thus be considered to be adverse to cartilage nutrition because: 1) it results in continuous contact over a certain area of the opposing cartilage surfaces (thus no pump will be operative and the rate of diffusion will be considerably decreased); and 2) there is no agitation of the synovial fluid in the joint cavity and hence the rate of diffusion will be lower. It is pertinent to observe that under normal circumstances prolonged immobility does not occur and is only imposed on human synovial joints by pathology or by therapy.

\section{CONCLUSIONS}

1. We have shown that the permeability of cartilage is the same in necropsy specimens as in the living animal. We have concluded that studies of material transport into cartilage carried out on necropsy specimens validly reflect in vivo conditions.

VOL. 50 B, NO. 1, FEBRUARY 1968 
2. We have studied the effect of agitation of the fluid in which cartilage is immersed upon the rate of diffusion of substances into cartilage and have found that agitation increases the rate of penetration up to three or four fold. We believe that it may be inferred from this fact that the nutrition of cartilage is partly dependent on joint movement.

3. We have examined the permeability of the bone-cartilage interface to water and solutes and have found that in the adult no detectable material transfer occurs across this zone. In the child on the other hand the bone-cartilage interface appears to be permeable to water and solutes.

4. We have measured the diffusion coefficient of glucose in cartilage and have hence estimated the depth of cartilage which can be adequately supplied with glucose from the synovial fluid in the presence and absence of agitation.

5. We have examined both experimentally and theoretically the possible effect of intermittent loading on the rate of penetration of substances into cartilage. We have concluded that at low pressures intermittent loading contributes little to the material transfer into cartilage. At high pressures intermittent loading does lead to the transport of solutes into cartilage but it cannot significantly increase the rate of transfer above that attributable to normal diffusion. Loading cartilage surfaces for prolonged periods of time without allowing intermittent relaxation would be expected to lead to decreased diffusion, without any absorption of fresh fluid attributable to the action of a pump, and would thus result in an overall decrease in the rate of penetration of substances into cartilage.

\section{NOMENCLATURE}

$\mathrm{C}=$ concentration of solute, moles per cubic centimetre.

$\mathrm{Co}=$ concentration of solute in the bulk solution, moles per cubic centimetre.

$\mathrm{C}_{i}=$ concentration of solute in the membrane at the solution-membrane interface, moles per cubic centimetre.

$\frac{d \mathrm{C}}{d x}=$ concentration gradient of solute, moles per cubic centimetre per centimetre.

$d=$ depth of penetration of solute into cartilage, centimetres.

$\mathrm{D}=$ diffusion coefficient, square centimetres per second.

$h=$ cartilage thickness, centimetres.

$d h$

$\frac{d h}{d t}=$ rate of decrease of cartilage thickness, centimetres per second.

$\mathbf{J}_{\mathbf{A}}=$ flux of solute $A$, moles per second per square centimetre of surface.

$p=$ pressure, grammes per centimetre per square second.

$\mathbf{P}=$ permeability coefficient, square centimetre per second.

$\mathbf{R}=$ radius of cartilage area under load, centimetres.

$\delta_{t}=$ tangential permeability, cubic centimetres seconds per gramme.

\section{REFERENCES}

Brower, T. D., AKahoshi, Y., and Orlic, P. (1962): The Diffusion of Dyes Through Articular Cartilage in Vivo. Journal of Bone and Joint Surgery, 44-A, 456.

Bywaters, E. G. L. (1937): The Metabolism of Joint Tissues. Journal of Pathology and Bacteriology, $44,247$. Collins, D. H. (1949): The Pathology of Articular and Spinal Diseases. London: Edward Arnold \& Co.

Denham, R. A. (1959): Hip Mechanics. Journal of Bone and Joint Surgery, 41-B, 550.

Exноlм, R. (1951): Articular Cartilage Nutrition. Acta Anatomica, Supplementum 15.

Екноцм, R. (1955): Nutrition of Articular Cartilage. A Radioautographic Study. Acta Anatomica, $24,329$.

Evans, E. B., Eggers, G. W. N., Butler, J. K., and Blumel, J. (1960): Experimental Immobilization and Remobilization of Rat Knee Joints. Journal of Bone and Joint Surgery, 42-A, 737.

HeLFFERICH, F. (1962): Ion Exchange. London: McGraw-Hill Book Company, Inc. 
Inman, V. T. (1947): Functional Aspects of the Abductor Muscles of the Hip. Journal of Bone and Joint Surgery, 29, 607.

Kantor, T. G., and Schubert, M. (1957): The Difference in Permeability of Cartilage to Cationic and Anionic Dyes. Journal of Histochemistry and Cytochemistry, 5, 28.

Kempson, G. E., Swanson, S. A. V., and Freeman, M. A. R. (1967): Stiffness Variations in Femoral Head Articular Cartilage. In Digest of The Seventh International Conference on Medical and Biological Engineering, Stockholm, Sweden, August 14-19 p. 508. Stockholm: Almqvist \& Wiksell.

McCutchen, C. W. (1962): The Frictional Properties of Animal Joints. Wear, 5, 1.

McKibin, B., and Holdsworth, F. W. (1966): The Nutrition of Immature Joint Cartilage in the Lamb. Journal of Bone and Joint Surgery, 48-B, 793.

Maroudas, A. (1968): Physico-Chemical Properties of Cartilage in the Light of Ion-exchange Theory. Biophysical Journal (in Press).

Ogston, A. G., and Sherman, T. F. (1961): Effects of Hyaluronic Acid upon Diffusion of Solutes and Flow of Solvent. Journal of Physiology, 156, 67.

Perry, J. H., ed. (1950): Diffusion in Liquids. In Chemical Engineer's Handbook. Third edition, p. 540. New York, Toronto, London: McGraw-Hill Book Company, Inc.

Ropes, M. W., and Bauer, W. (1953): Synovial Fluid Changes in Joint Disease. Cambridge, Mass.: Harvard University Press.

Rydell, N. W. (1966): Forces Acting on the Femoral Head-prosthesis. Acta Orthopaedica Scandinavica, Supplementum 88 .

VOL. $50 \mathrm{~B}$, NO. 1, FEBRUARY 1968 